\title{
ACCESIBILIDAD A LOS CENTROS POBLADOS EN EL VALLE DEL ITATA, PROVINCIA DE ÑUBLE, CHILE.
}

\author{
Christian LOYOLA GÓMEZ \\ chloyola@ubiobio.cl \\ Juan RIVAS MALDONADO \\ jrivas@ubiobio.cl
}

Recibido: 04/03/2014

Aceptado: 01/12/2014

RESUMEN: Se analiza la accesibilidad de los centros poblados del Valle del Itata, en base a las características de su infraestructura vial, considerada para el caso como una red compuesta por nodos y aristas, de esta forma posible de equiparar a un grafo matemático haciendo posible la medición mediante el uso de sistemas de información geográfica y la aplicación de modelos existentes con los que se establecen zonas de mayores y menores niveles de accesibilidad de los nodos. Se destaca que a pesar de la aplicación de diferentes índices, los centros poblados con mayor accesibilidad se repiten a lo largo del estudio, estos lugares gozan de cierta centralidad y responden a restricciones geográficas y a la impedancia del desplazamiento.

PALABRAS CLAVE: Grafo, Accesibilidad Real, Accesibilidad Ideal, Accesibilidad Absoluta, Accesibilidad Relativa.

THE ACCESSIBILITY TO THE TOWNS IN THE VALLE OF ITATA, ÑULE PROVINCE, CHILE.

ABSTRACT: The accessibility of the towns of Valle del Itata is analyzed based on the characteristics of its roadway infrastructure, considered for this case as a network composed by nodes and edges, making it possible to equate to a mathematical graph allowing to perform the measurements using geographic information systems and the application of existing models to establish areas of minor and major accessibility node levels. It is emphasized that despite the application of different rates, the populated centers with greater accessibility are repeated throughout the study, these places have a certain centrality and respond to geographical constraints and the impedance of displacement.

KEYWORDS: Graph, Real Accessibility, Ideal Accessibility, Absolute Accessibility, Relative Accessibility. 


\section{INTRODUCCIÓN}

Se aborda el análisis espacial buscando dar explicación y posibilidad de previsión con respecto al estado y la evolución probable de los objetos/unidades geográficos, considerando su situación en relación con los otros objetos geográficos. El análisis propuesto está dado entonces por las interacciones horizontales entre los lugares, en virtud de la espacialización de la superficie terrestre por las sociedades humanas y la distribución de sus actividades para el desarrollo. Para ello el modelo de análisis espacial cuenta con una serie de índices que buscan resumir las estructuras del sistema geográfico mediante el uso de sistemas de información geográfica.

Cabe considerar que existen cuatro factores que determinan el desarrollo de una región: las infraestructuras, la localización, la aglomeración, la estructura de asentamientos y la estructura sectorial de la economía. El más influyente de ellos es la infraestructura de transporte ya que reduce o aumenta la distancia, la cual por una parte frena las interacciones y por otra hace variar el valor de los lugares en función de su situación geográfica, principalmente por los temas referidos a áreas de influencia o de localización de estructuras comerciales o industriales. Interviniendo en el flujo de personas y mercancías, afectando en forma directa el funcionamiento socioeconómico de espacios territoriales (Nogales et al., 2002), (CARDOZO et al., 2009).

Se produce de esta forma una zona heterogénea y anisotrópica, constituida por nodos y ejes jerarquizados que organizan los flujos de circulación en territorios desigualmente estructurados (GuTiérRez, Monzón, 1993). Que sumados al conjunto de elementos materiales, tales como: las infraestructuras; inmateriales: ondas o informacionales, aseguran la relación de diferentes lugares de un territorio y de las entidades que lo ocupan, estableciendo un red. Para BosQue (1997) es "un sistema interconectado de elementos lineales, que forman una estructura espacial por la que pueden pasar flujos de algún tipo" (Bosque, 1997: 207). Ramírez (2003) basado en Comas y Ruíz, en la misma línea, define la red como un sistema interconectado de líneas por las que se desplazan una serie de elementos: personas, bienes, recursos u otros, que se conectan con nodos que corresponden a cruces de segmentos.

La red está compuesta entonces, por elementos lineales, permanentes o temporales y por elementos nodales necesarios para la organización de flujos y para el funcionamiento del sistema en el que está inserta la red. De los elementos lineales (aristas) el mejor ejemplo: son las vías de circulación, y de los elementos nodales los núcleos de población, su consideración incorpora atributos o características. LuPIEn, Moreland y Dangermond, (1987), en Bosque (1997) caracterizan el análisis 
de redes, como aquel en que la existencia de las redes obedece a una necesidad de movilidad, de comunicación, de intercambio debido a lo heterogéneo del espacio geográfico al igual que BARBERo y QuinN (1986), en CARDOZo et al., (2009).

El atributo temático más importante asociado a las aristas de una red es la longitud o coste de recorrerla, la determinación de su longitud en una recta es a través de la definición de distancia euclidiana para, a partir de las coordenadas de sus nudos (de origen y final), determinar su longitud. La distancia es fundamental en la aplicación de cualquier indicador de accesibilidad, aunque no es el único factor, sí permite plantear y resolver un amplio conjunto de problemas prácticos como son: la determinación de rutas óptimas para vehículos que deben moverse en una red de carreteras, la localización de servicios e instalaciones (bomberos, policías, hospitales, comercio) de modo óptimo en cuanto a costes de recorrido para su empleo por los usuarios, la delimitación de distritos y áreas de influencia. Ahora bien, la asignación de caminos de distribución de un producto debe valorar el peso que tiene en el uso o no que haga una población de un servicio y por lo tanto del nivel de bienestar que este podría dar a los usuarios (SALADO, 2004).

Una buena infraestructura de transporte permite que nuevos espacios sean accesibles para la actividad humana, de forma más rápida y en una gran escala de forma suficiente y eficiente, Chile se caracteriza por poseer una red vial cuya longitud total es de 80.528 kilómetros, de ellos unos $17.269 \mathrm{~km}$. se encuentran pavimentados que han sido clasificados como: caminos nacionales, caminos regionales y caminos comunales. Para “(...) superar la fricción impuesta por la distancia que separa a la población de los diversos centros de actividad" (BARBERO y QUINN, 1986) existe una política nacional de infraestructura orientada a concesionar mediante licitación, la pavimentación correspondiente a anillos de circunvalación y vías de acceso de los centros de producción y centros urbanos más poblados (CEPAL, 2012).

Como las carreteras conforman una red y por lo tanto existe más de una arista, para encontrar un resumen de las longitudes de todas ellas existen diversos índices que proporcionan una valoración cuantitativa (Bosque, 1997). La construcción de esta red supone que las líneas sinuosas y complicadas que unen nudos de la red se sustituyan en un grafo por líneas rectas estableciendo la relación de sinuosidad o relación entre la longitud real de una línea y su longitud si fuese recta. La introducción de grafos en Geografía corresponde a Garrison y Marble en los años '60; a partir de ese periodo la Teoría de Grafos en Geografía puede mencionar a Haggett y Chorley (1969), Tinkler (1977), Potrikovsky y TAYlor (1984), Seguí Pons y Petrus Bey (1991), Bosque (1997) y Rodríguez (2006) (Insaurralde y Cardozo, 2010). 
Un segundo elemento de análisis corresponde a la cohesión topológica de la red para la medición de la facilidad o dificultad de los flujos y movimientos en su interior. La longitud de las aristas se relaciona con las dificultades de flujo, es decir: mientras más extensa mayor dificultad de flujo. Asimismo el número de interconexiones es asociado con la facilidad de flujo, es decir: mientras más interconectado mayor facilidad de desplazamiento al interior de la red. Para ello es importante contabilizar el número de aristas de la red en relación al número de nudos que existen, lo que proporciona una medida de conectividad o cohesión (BosQue, 1997).

\section{I.1 Accesibilidad}

El concepto de accesibilidad no posee una única y consensuada acepción, ya que es entendida en términos geométricos (cercanía-lejanía), económicos y sociales o también desde la perspectiva de "facilidad con que un servicio puede ser alcanzado desde una localización" (SALADO, 2004:21). GoodALL (1987) define la accesibilidad como la facilidad con que se puede llegar a un sitio desde otras localidades; Deichmann (1997) trata de ofrecer una definición más amplia, como facilidad de acceso a oportunidades económicas y sociales. Naturalmente, cuanto mejor es la accesibilidad, más competitivos y por tanto más exitosos en términos de crecimiento económico son los territorios (LINNEKER, 1997), con independencia de otros factores. Esto ha llevado al intento de clasificar los distintos usos y componentes del término accesibilidad, proponiéndose:

1. según los elementos contemplados en el problema

2. según el tipo de espacio, métrica, usos del suelo y grupos sociales

3. según las características del servicio a evaluar

4. según las características de la demanda

5. según las características del modelo de accesibilidad empleado (SALADO, 2004).

$\mathrm{Al}$ analizar cada uno de estos componentes es muy difícil llegar a una única definición, también cuestionable, ya que dependiendo de los objetivos y situaciones particulares, cada una de ellas puede ser útil según los objetivos y elementos considerados en cada caso. En esta circunstancia los SIG constituyen una potente herramienta por su capacidad de manipular y superponer grandes volúmenes de datos georreferenciados, incorporando distintos momentos temporales como métodos de análisis y visualización del territorio (FARrow y Nelson, 2001), (SAlAdo, 2004).

Es así como la accesibilidad de un lugar, en términos generales se puede definir como la mayor o menor facilidad con la cual un lugar puede ser alcanzado a par- 
tir de uno o varios otros lugares, por uno o varios individuos susceptibles de desplazarse con la ayuda de todos o algunos de los medios de transporte existentes. Bosque (1997) citando a Osberg (1976) señala que la accesibilidad es una medida relativa en función de la facilidad de acceso de un punto del espacio sobre un hecho. Estas dos definiciones tienen implícito un importante elemento que es el de la distancia, entendida como el intervalo a franquear para ir de un lugar a otro, teniendo una significación de separación su recorrido necesita un esfuerzo, un gasto de energía.

En el sentido de la equidad social la accesibilidad se vuelve fundamental para la localización e instalación de cualquier equipamiento ya que en su mayoría son financiados por la administración pública, por lo tanto deben tener iguales o similares condiciones de acceso para toda la población de un territorio (Bosque, 1997). De igual modo, Salado (2004) plantea que en la planificación territorial los equipamientos colectivos cumplen un rol relevante en el desarrollo regional, ya que las actividades económicas y el bienestar social se ven beneficiados por una adecuada dotación de equipamientos colectivos.

El análisis de la accesibilidad puede ser usado entonces, en políticas de desarrollo (FARROW y NelSON, 2001) puesto que una baja accesibilidad refleja como indicador carencias en los sectores, traduciéndose por tanto como indicador de factores de Desarrollo (Gross, 1998). Debido a lo anterior intervenciones en la infraestructura de transporte modificarían las condiciones de accesibilidad y por ende potenciarían espacios no desarrollados (GutiérReZ y Monzón, 1993).

Desde esta perspectiva, la provincia de Ñuble viene siendo propuesta por un importante número de actores sociales, como una futura región, lo que le otorgaría niveles de autonomía, facilitando su desarrollo. Para ello se hace necesaria una sistematización de las características estructurales, potencialidades y debilidades de sus territorios (LiRA, 2006). Relevante es entonces conocer cuáles son los niveles de accesibilidad entre los centros poblados y los centros de actividad económica en el territorio denominado Valle del Itata de la provincia de Ñuble.

\section{AREA DE ESTUDIO}

\section{II.1 Descripción del área de estudio}

Chile se extiende a lo largo de más de $4.000 \mathrm{~km}$ en una franja entre la Cordillera de los Andes y la costa suroriental del Océano Pacífico. A partir de 2007 el país cuenta con 15 regiones, 54 provincias y 346 comunas en total. La población está fuertemente concentrada en la zona centro del país, en la región 
metropolitana, donde se encuentra Santiago, la capital. Las ciudades que le siguen en importancia son Valparaíso y Concepción, esta última en la región del Biobío, los extremos del país se hallan menos densamente poblados. Las principales actividades económicas del país son los servicios financieros y la industria manufacturera, dirigidas principalmente al consumo interno, siendo en cambio la minería la principal actividad exportadora.

De acuerdo a la división política-administrativa de Chile, el área de estudio se ubica en la Región del Biobío, Provincia de Ñuble, el territorio del Valle del Itata está compuesto por los municipios de Cobquecura, Trehuaco, Coelemu, Portezuelo, Ránquil, San Nicolás y Quillón, integrándose en el año 1996 los municipios de Ninhue y Quirihue. Sus límites son: al norte, la región del Maule con sus comunas de Parral y Cauquenes; al sur, las comunas de Florida y Tomé; al este, las comunas de Chillán y Bulnes; al oeste, el Océano Pacifico.

Geográficamente el área de estudio se ubica en el sector norponiente de la Región del Biobío, específicamente x1 689.738,43 km - x2 764.634,32 km y y1 $5.909 .372,09 \mathrm{~km}-\mathrm{y} 26.013 .540,61 \mathrm{~km}$. Su emplazamiento presenta sectores sobre tres grandes unidades del relieve regional, las plataformas litorales (4,62\%) de limitada extensión, la Cordillera de la Costa $(62,67 \%)$, con altitudes relativamente bajas y disectada en dos cordones que rodean la cuenca de Quirihue, y la Depresión Intermedia (21,94\%) (ReBolledo et al., 1994).

Las cuencas relevantes son las del río Ñuble y el río Itata, además de los ríos Lonquén, Changaral y Coyanco. El curso del río Itata estructura, un valle de dirección este-oeste de significativa relevancia en la morfología natural del territorio (MOP, 2004). La Cordillera de la Costa, pese a su altitud relativamente baja (400 msnm) provoca diferenciación topoclimática entre sus vertientes: con mayor cantidad de precipitaciones (la occidental) y su vertiente oriental con mayor aridez. La pluviometría varía entre los 1.000 y $1.300 \mathrm{~mm}$ anuales (fundamentalmente en los meses de junio a septiembre) (Henríquez, 1990).

Las nueve comunas del Valle del Itata cubren una superficie de 3.660,10 $\mathrm{km}^{2}$ y contienen una población de 80.272 habitantes, de los cuales el $51,24 \%$ son hombres y un $48,76 \%$ son mujeres, presenta una densidad de $21,5 \mathrm{hab} / \mathrm{km}^{2}$ (INE, 2008), densidad que es superior a la nacional, $\left(20\right.$ habitantes $\left./ \mathrm{km}^{2}\right)$. La población se ubica en un 44,87\% en el área urbana y un 55,13\% en el área rural. Respecto del territorio, su superficie corresponde al $28 \%$ de la Provincia de Ñuble y al 9,9\% de la Región del Biobío. La comuna de Quirihue es la de mayor tamaño $\left(589 \mathrm{~km}^{2}\right)$, seguida de la comuna de Cobquecura $\left(570,3 \mathrm{~km}^{2}\right)$. Las de menor superficie son las comunas de Ránquil $\left(248,3 \mathrm{~km}^{2}\right)$ y Portezuelo $\left(282,3 \mathrm{~km}^{2}\right)$. 
El territorio se ubica en una región que en términos globales tiene características muy diferentes, principalmente por el peso urbano de la intercomuna Concepción-Talcahuano y los roles de cada una de sus provincias. Constituyendo un territorio de alta ruralidad y mayoritariamente silvo-agropecuario en su actividad económica (Henríquez, Olivares, 1997) la cual absorbe el 41\% de la fuerza de trabajo. El territorio es conocido por el desarrollo de viñas (Del Pozo, 1999) a las cuales se les otorga gran relevancia (Alvarado, 2003), (Couyoumdian, 2006) es así como la denominación de origen ${ }^{1}$ Valle del Itata (ÁlLVAREZ, 2001), está vinculada a este tipo de producción, su importancia es tal, que opaca (nominalmente) el desarrollo silvícola para la alimentación de la planta de Celulosa Nueva Aldea (originalmente Celulosa Itata) existente en la zona.

\section{METODOLOGÍA}

\section{III.1 Descripción}

Bosque (1997) señala que el análisis de dotación de infraestructuras puede obtenerse comparando medidas físicas de las mismas con la población o la superficie de un determinado territorio. De esta forma los fenómenos de la realidad son esquematizados representando una malla de carreteras y caminos que serán consideradas aristas y cuyas ciudades y pueblos serán considerados nodos, al igual que los cruces e intersecciones de caminos. Los datos estarán completados con el indicador de población según Censo 2002, con el nombre del municipio y las coordenadas geográficas dadas por la digitalización (UTM, WGS 1984 HUSO $18 \mathrm{~S}$ ). En este caso la selección corresponde a entidades urbanas con más de 200 habitantes, esta selección es en consideración a que el Instituto Nacional de Estadísticas (INE) tiene como primera entidad a los caseríos, cuya clasificación cuenta con más de 300 habitantes, lo que deja fuera a un número importante de localidades. La caracterización se realizará al equiparar la red a un grafo matemático indicando el nodo de origen y el nodo de destino y por las relaciones presentadas entre los nodos, para la posterior elaboración de una Matriz de Conectividad donde se indica con 1 las relaciones directas entre nodos y con 0 la

${ }^{1}$ Es un tipo de indicación geográfica aplicada a un producto agrícola o alimenticio cuya calidad o características se deben fundamental y exclusivamente al medio geográfico en el que se produce, transforma y elabora. Las indicaciones geográficas y las denominaciones de origen protegen productos originarios del país o de una región o localidad, siempre que tengan una calidad, reputación u otra característica imputable a su origen geográfico. En el caso de las denominaciones de origen, deben presentar además factores naturales y humanos que inciden en la caracterización del producto. 
ausencia de una conexión directa (BosQue, 1997) además de construir una Matriz de Accesibilidad Topológica.

Para valorar la accesibilidad que la red confiere en diversos puntos del territorio Nastagi (2003) propone la comparación de la relación existente en tres tipologías de redes. Sin embargo Bosque (1997) indica la comparación entre dos de ellas solamente: la red real representada por el sistema de carreteras y caminos entre cada par de nodos, lo que permite determinar el resumen de las longitudes de las aristas de la red, y la red ideal o ficticia formada por la distancia entre cada par de nodos, es decir: la longitud del segmento que los une. Mierez (2004) y Gómez (2008) en Martínez (2012) plantean que para la visualización de la funcionalidad de la malla vial se requieren los siguientes índices:

- Índice de Densidad media $\left(I_{S}\right)$ : Con esta medida se puede inferir el nivel de desarrollo de la configuración vial ya que se asocia un mayor desarrollo en las áreas con más kilómetros de vías, se expresa como la relación entre la longitud en $\mathrm{km}$ de la red vial (L) y la superficie en $\mathrm{km}$ del área de estudio (S):

$$
I_{S}=\mathrm{L} / \mathrm{S}
$$

- Coeficiente de Engels $\left(I_{E}\right)$ : permite obtener la eficacia vial comprobando la facilidad de circulación de personas e intercambio de bienes y servicios. Para estos casos a mayor valor, mayor es la eficiencia vial. $\mathrm{Su}$ cálculo es a través de la relación de la longitud de las vías $\left(\mathrm{km} \mathrm{v}^{*} 100\right)$, tanto con la superficie del área estudiada (S) como con el número de habitantes que alberga $(\mathrm{P})$ :

$$
\mathrm{I}_{\mathrm{E}}=\mathrm{km} \mathrm{v} * 100 / \sqrt{ }(\mathrm{S} * \mathrm{P})
$$

- Accesibilidad Ideal $\left(A I_{1}\right)$ : permite observar cuanta distancia (di) es la que existe entre un punto con respecto a cada punto (n) del área de estudio. Se calcula a partir de la sumatoria de las distancias lineales entre un punto con respecto a otros

$$
A I_{1}=\sum \mathrm{di}_{1 \mathrm{n}}
$$

- Accesibilidad Real $\left(A R_{\gamma}\right)$ : permite precisar la distancia a través de las carreteras y caminos, entre un punto del espacio respecto a otros puntos, para calcularla

$$
A I_{1}=\sum \mathrm{di}_{1 \mathrm{n}}
$$

- Índice de la Calidad de la Comunicación (ICC): para el cálculo de este índice se comparan las distancias ideales con las reales a través del co- 
ciente de los índices de accesibilidad ideal y real respectivamente, con el fin de comprobar qué tan cercano es lo ideal de lo real:

$$
I C C_{1}=A I_{1} / A R_{1}
$$

- Índice de Trayectoria (IT): este índice indica el porcentaje de longitud extra recorrido para llegar de un punto a otro, de no recorrerlo en línea recta. Se calcula mediante el cociente de los índices de accesibilidad real e ideal y se obtienen valores oscilantes al uno (1), indicando que cuanto mayor sea la trayectoria a recorrer, mayores serán los valores.

$$
I T_{1}=A R_{1} / A I_{1}
$$

\section{III.2 Conectividad o Cohesión}

El análisis simultáneo de todas las aristas y nodos de la red, determinan la cohesión de la red (Bosque, 1997), la relación del número de aristas con el número de nodos proporcionan la medida de conectividad, es decir: el grado de interconexión del circuito (Seguí y Garrido, 1986; Cantó y otros, 1988) la mayor o menor complejidad estructural de la red, está ligada directamente al número de nodos, aristas y a su disposición espacial (Seguí Pons y Petrus Bey, 1991). En este caso mientras más aristas tengan el grafo, mayor será el grado de conectividad (Kansky, 1963 en GarRido 1995) (Insaurralde, Cardozo, 2010). Para realizar las mediciones existen índices de conexión, entre los cuales se encuentran los siguientes:

- Índice Beta: expresa la relación entre las aristas y nodos de un grafo. Donde (a) corresponde a las aristas y (n) a los nodos.

$$
\beta=\mathrm{a} / \mathrm{n}
$$

- Índice Gama: relaciona el número de aristas existentes y el número máximo que puede existir dentro de un grafo determinado, teniendo en cuenta la cantidad de nodos de una red.

$$
\gamma=(2 * a) /(n *(n-1))
$$

- Número Ciclomático (Índice $\mu$ ): determina la cantidad de circuitos que existen dentro de un grafo (Bosque, 1997). Entendiendo por circuito, cada una de las múltiples maneras que existen para ir desde un nodo hasta el mismo, sin tener que pasar dos veces por el mismo arco. Se calcula restando el número total de aristas (a) al número de nodos necesario para formar un árbol (n-1), restándose además los grafos inconexos 
(g) que puedan existir ya que la red no es coherente (GARRIDO, 1995); (InsurRaLde y CARDOZO, 2010).

$$
\mu=\mathrm{a}-(\mathrm{n}-1)-\mathrm{g}
$$

- Índice Alfa: relaciona el número de circuitos observados en el grafo $(\mu)$ y los circuitos que existirán en caso de tratarse de un grafo completo. Su valor resultante varía entre 0 para un grafo sin ningún circuito y 1 para un grafo completo (Bosque, 1997).

$$
\alpha=\mu /(2 * n-5)
$$

\section{III.3 Accesibilidad}

La realización de las medidas de accesibilidad fue establecida considerando que el sistema de infraestructura vial es un sistema cerrado, marcando su límite en el territorio del Valle del Itata y que los núcleos de población son nodos jerarquizados medidos según la facilidad de acceso desde cada uno en relación a los restantes nodos del grafo (GARrido, 1995 en Insaurralde y Cardozo, 2010). Determinándose la Accesibilidad, tanto topológica como absoluta, a través de una estructura vectorial, principalmente para el cálculo de distancias entre objetivos.

Con la creación de una Matriz de Accesibilidad Topológica, se señalan la mínima cantidad de aristas necesarias de recorrer entre un nodo y cada uno de los restantes (Seguí Pons y Petrus Bey, 1991 en Insaurralde y Cardozo, 2010), esta matriz permite obtener:

- El Número Asociado de un Nodo (NS) (Bosque, 1997), es decir: el número mínimo de aristas que es necesario recorrer para unir un nodo con el otro más distante topológicamente.

- Número Asociado de Köning que resulta ser el valor mayor de cada fila de aristas que es necesario recorrer para unir un nodo con el otro más distante topológicamente.

- Índice de Shimbel, que se obtiene sumando los valores de cada fila, es decir: mostrando el número de aristas por atravesar para ir desde un nodo a todos los demás de la red. Donde $d x y$ es el número de aristas que separa a los nodos x e y por el tramo más corto. (GARRIDO, 1995) lo expresa como:

$$
\mathrm{Ay}=\sum d x y
$$

Con el Índice de Shimbel es posible determinar otros tres índices, el primero de ellos denominado: 
- Índice $G$ de Dispersión que permite lograr una visión de conjunto del grafo en cuanto a la accesibilidad, su cálculo es a través de la suma de todos los Índices de Shimbel de cada nodo. Del mismo modo es posible obtener la accesibilidad media de la red, dividiendo el índice de dispersión entre el número de nodos (BOSQUE, 1997); (GARRIDO, 1995); (CARDOZO et al., 2009).

- Índice de Accesibilidad Media (PotryKowsky, 1984); (GARrido, 1995); (CARdozo et al., 2009); (Insaurralde y CARdozo, 2010), se trata de un cociente entre la accesibilidad topológica (Ay) y el número total de nodos (n), expresada de la siguiente manera:

$$
P y=A y / \mathrm{n}
$$

- Índice Omega: sirve para obviar la dificultad de comparar redes con distinto número de nodos, siendo Amáx. el índice de Shimbel más alto, y Amín. El más bajo. Expresado como:

$$
\Omega y=(A y-A m i ́ n) 100 / A m a ́ x-A m i ́ n
$$

\section{III.4 Accesibilidad Absoluta y Relativa}

Con el indicador de accesibilidad absoluta se calcula el promedio de las impedancias que separan a cada nodo con respecto de los diferentes centros de población a través de la red (GuTiÉrRez y Monzón, 1993) (por el camino de mínima impedancia) considerando a la cantidad de habitantes como factor de ponderación. Esta accesibilidad no está solo condicionada por la dotación de infraestructuras de transporte sino que también se va ver influida por la situación geográfica del área. Para el cálculo de la impedancia a través de la red, se ha utilizado el tiempo promedio de circulación por las diferentes carpetas de rodado lo que hace variar los promedios de circulación (Tabla $\mathrm{N}^{\circ} 1$ ). Sin embargo se ha hecho el promedio de las tres dividido por la distancia para obtener una calificación de tiempo comparada con la realidad.

Tabla $\mathbf{N}^{\circ} 1$ Impedancias

\begin{tabular}{|l|l|}
\hline & Velocidad Promedio \\
\hline Asfalto / Cemento & $70 \mathrm{~km} / \mathrm{hr}$. \\
\hline Ripio & $50 \mathrm{~km} . / \mathrm{hr}$. \\
\hline Tierra & $30 \mathrm{~km} . / \mathrm{hr}$. \\
\hline
\end{tabular}

Elaboración propia

En el actual caso la accesibilidad se expresa en términos de tiempo de viaje. En este sentido el tiempo de viaje es calculado de acuerdo al promedio de circulación 
asignado a cada nodo de acuerdo a la distancia vial o real (Accesibilidad Absoluta o Accesibilidad Relativa) del nodo de destino, adaptando el procedimiento propuesto Farrow y Nelson (2001) en PABLo et al., (2002), luego se reescala mediante la ecuación para expresarla en términos de tiempo requerido en cruzar de un nodo a otro.

$$
S=d *\left(\frac{1}{\operatorname{vel} *\left(\frac{1000}{3600}\right)}\right)
$$

Expresando el cálculo del índice de la siguiente forma:

$$
I A A_{i}=\frac{\Sigma\left(H_{j} * S_{a}\right)}{\Sigma H_{j}}
$$

Donde $I A A_{i}$ es la accesibilidad absoluta del nodo $i, H_{j}$ la cantidad de población de la localidad de destino, $S_{\mathrm{a}}$ el tiempo de viaje real que considera las impedancias, establecidas de acuerdo a los tiempos mínimos de desplazamiento a través de la red de carreteras. Con este indicador que se obtuvo se generó un mapa temático de Accesibilidad Absoluta. Se clasificó la información en intervalos determinando clases de accesibilidades desde niveles muy bajos hasta muy altos, los cuales se representaron mediante cartografía temática.

Para evitar los efectos de la localización geográfica de los distintos nodos, se ha propuesto un Indicador de Accesibilidad Relativa, en la cual la accesibilidad únicamente va a depender de la dotación de infraestructuras. Neutralizando el efecto de la localización geográfica, con el objetivo de resaltar los efectos de red de caminos y carreteras sobre la accesibilidad, reflejando la característica geométrica de la red y el tipo de infraestructura hacia los distintos centros poblados, relacionando el tiempo de viaje real con el tiempo de viaje ideal, que se originaría por el recorrido en una autopista pavimentada y en forma de línea recta (Analistas Económicos de Andalucía, 2001 en Ávila, 2012).

$$
I A R_{i j}=\frac{\sum\left(H_{j} * S_{a}\right)}{\Sigma\left(H_{j} * S_{r}\right)}
$$

Donde IARij sería la impedancia mínima ideal entre el nodo $i$ y el nodo $j$, es decir, el tiempo de acceso considerando la existencia de la mejor infraestructura disponible. De este modo, para cada relación se determina el tiempo de viaje mínimo de un centro poblado al centro más próximo. Midiendo la proximidad de cada centro poblado y su facilidad para desplazarse hacia el nodo más cercano (López et al., 2008), cuando el resultado tienda a 1 la impedancia real estará cerca de la ideal, pero irá aumentando a medida que la primera se aleje de la segunda. 


\section{RESULTADOS}

\section{IV.1 Descripción de las rutas}

La red vial del territorio tiene dos importantes vías con dirección oeste (borde costero), una que considera características turísticas y la otra, características productivas, en función de rutas para el transporte de madera. La red vial está compuesta por cuatro tipos de carpeta, en primer término el asfalto que ocupa un $10,35 \%$ del total de la red, el ripio que representa poco más de la mitad de la red con un 51,36\%; una mezcla de ripio y tierra que a pesar de no representar más de un uno por ciento es relevante por la discontinuidad en la calidad de las carpetas de acceso a los centros poblados, y por último la tierra que con $37,32 \%$ de la red, es particular en su consideración dentro del territorio, por la gran cantidad de kilómetros.
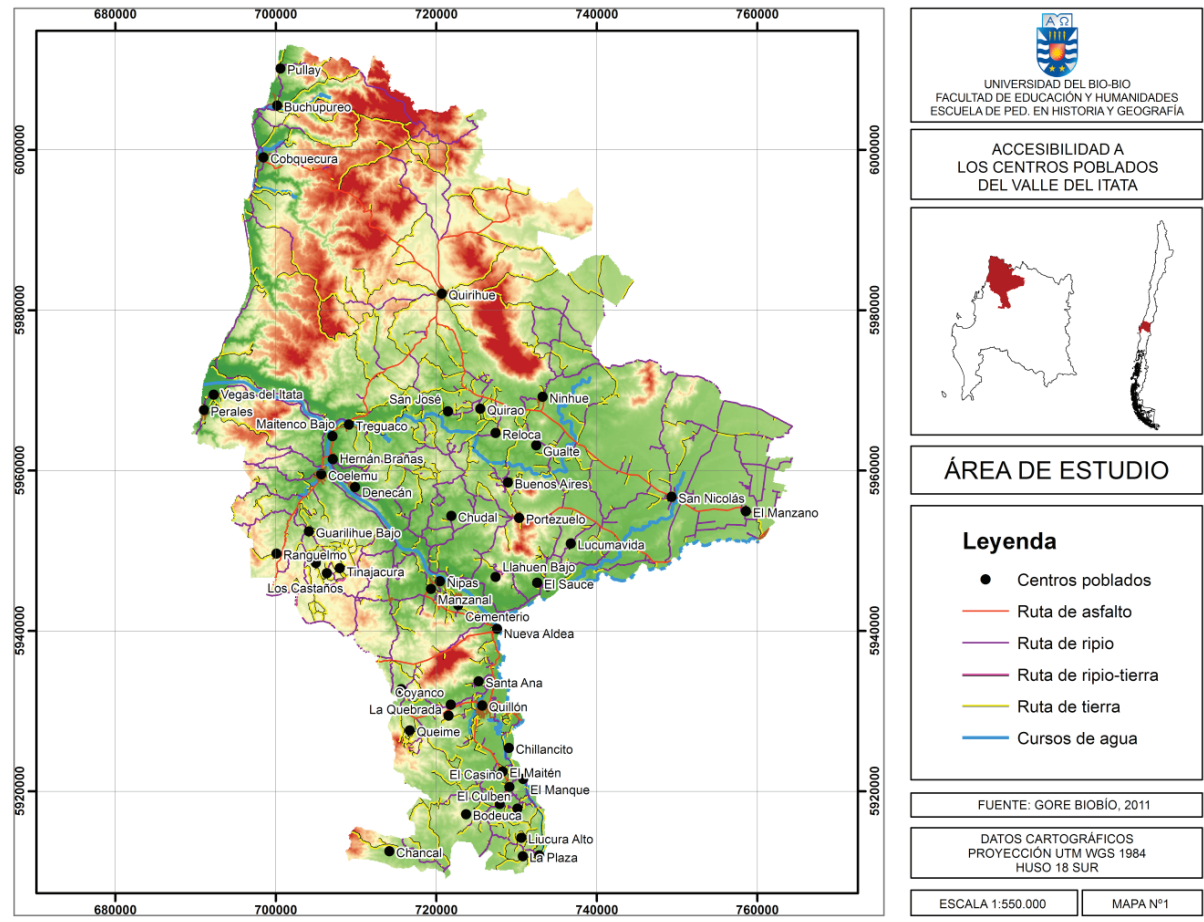

La densidad vial representa la relación entre el área utilizada por kilómetro de vía. Al emplear el Índice de Densidad Media: $\mathrm{I}_{\mathrm{s}}=3394 \mathrm{~km} / 3660,2 \mathrm{~km}^{2}$ sus resultados implican que la densidad vial es de $0,927 \mathrm{~km} / \mathrm{km}^{2}$, es decir: por cada $\mathrm{km}^{2}$ existen menos de un kilómetro de vías, expresando a todas luces, un pobre desarrollo de la configuración vial. Si se sectoriza el territorio por comunas, el 
Índice de Engels demuestra que las comunas de Ranquil $(20,6)$ y Portezuelo $(17,4)$, presentan la mayor facilidad para la circulación de personas, el intercambio de bienes y el desarrollo de los servicios. En esta clasificación Ninhue $(16,6)$ y San Nicolás $(16,3)$ se estructuran con indicadores en la medianía de la tabla y el resto de las comunas se despliegan con limitadas facilidades para la circulación.

Los índices se calcularon para todos los nodos del territorio, a partir de la generación de las matrices de accesibilidad ideal y real, utilizando la herramienta proporcionada por SIG. Las matrices contienen las unidades espaciales, calculadas en los kilómetros existentes entre ellos. Los valores obtenidos tanto para la Accesibilidad Ideal (AI) como para la Accesibilidad Real (AR) se obtienen a partir de la sumatoria de las filas. Los casos de mayor accesibilidad ideal (AI) se refieren principalmente a las cabeceras comunales Coelemu (1664), Portezuelo (1417), Ninhue (1723) y Quillón (1492) y a localidades intermedias que se encuentran en el borde de los caminos, teniendo correspondencia con las mediciones de accesibilidad real (AR). Los nodos considerados menos accesibles corresponden a las localidades del norte del territorio e involucran a la cabecera comunal de Cobquecura (2888) y a los nodos más alejados respecto a los demás, el resto de los nodos se encuentra en la medianía de la tabla disminuyendo su accesibilidad a medida que se alejan del centro del territorio.

Los resultados del Índice de la Calidad de la Comunicación (ICC) e Índice de Trayectoria (IT) verifican que ningún nodo tiene coincidencia entre la distancia ideal y la real ya que ninguno se acerca al valor óptimo (1). Los nodos con mayor calidad de comunicación, corresponden a las cabeceras comunales de Ninhue $(0,71)$, Portezuelo $(0,71)$, Quillón $(0,75)$ y Quirihue $(0,68)$ y sus sectores aledaños, todos estos nodos se encuentran en zonas centrales del territorio. Respecto de las trayectorias a recorrer las cabeceras comunales presentan los menores índices Ninhue $(1,4)$, Portezuelo $(1,4)$, Quillón $(1,3)$ y Quirihue $(1,48)$. Cabe destacar que con este indicador, Coelemu $(0,58) ;(1,7)$ respectivamente, aparece alejado de las representaciones óptimas, coincide esto con las restricciones viales planteadas por la presencia del río Itata (en el borde sur), pero no con los resultados de otros índices.

\section{IV.2 Conectividad o Cohesión}

Por definición, los valores del Índice Beta que se encuentran sobre 1 dan cuenta de una red que tiende a complejizarse, en este caso el resultado del índice arroja 1,53 dando cuenta de una red en que se han construido algunos de los ca- 
minos posibles entre los centros poblados, lo que en un modelo como el de teoría de grafos se interpreta como el de idea de conectividad, sin embargo la realidad se estructura como un árbol invertido, teniendo en consideración que las estructuras viales se han ido adaptando al relieve en este caso la Cordillera de la Costa y a la presencia de cursos de agua tales como la desembocadura del río Itata.

El resultado del Índice Gama $(0,05)$ da cuenta que la cantidad de aristas que pueden existir en el grafo en relación con las que efectivamente existen, describen un sistema de centros poblados poco conectado y con un desarrollo de su conectividad que se ubica bajo la medianía de la tabla, considerando que cuando el índice es uno (1) se está frente a una red cuyo circuito está completamente conectado, algo que económicamente y desde el punto de vista práctico es inviable.
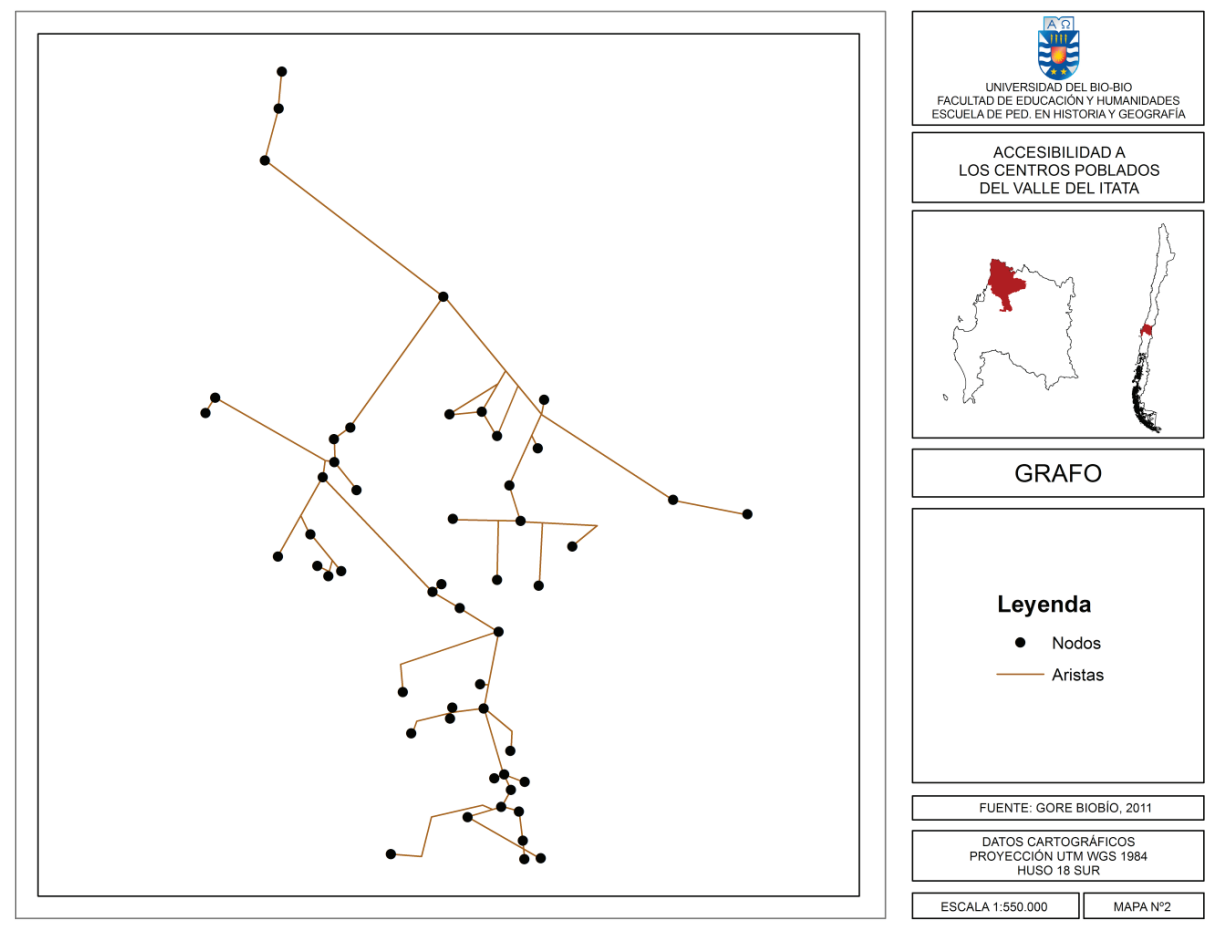

Cinco (5) es el Número Ciclomático o de circuitos que existen para ir desde un nodo hasta él mismo, sin pasar dos veces por la misma arista. Se evidencia que este número es limitado y nuevamente queda en evidencia que la forma de árbol invertido demuestra que existe una alta concentración de centros poblados unidos por un solo camino, y que la conectividad real, en el sentido 
estructural está conformada por caminos únicos entre centros poblados. Lo que equivale a un bajo grado de conexión del sistema y a inexistencia de un circuito integrado.

Finalmente el Índice Alfa relaciona el número de circuitos observados y los que existirían si se tratase de un grafo completamente conectado, comparando el valor del Número Ciclomático, como la cantidad de circuitos posibles, y el valor máximo que podría alcanzar, es decir: el total de circuitos por cantidad de nodos. En este caso el índice alcanza el valor 0,045 dando cuenta del desarrollo de la red, cuyo valor máximo para este tipo de índices es 1 evidenciando que la cantidad de conexiones existentes en relación con las posibles es limitada, no permitiendo el ir y volver a los distintos centros poblados por caminos diferentes.

\section{IV.3 Accesibilidad}

Respecto de la Accesibilidad los primeros indicadores (Números Asociados) establecen que las localidades de Coelemu, Vegas de Itata, Ranguelmo, Cementerio y Manzanal, tienen la mayor accesibilidad (11, 12, 12, 12 y 11 respectivamente). Cabe la consideración que todas ellas se encuentran al suroeste del territorio, a orilla de carretera presentando entradas y salidas diferenciadas pero que solo corresponden a un camino, por tanto lo que el modelo asume como nodos de mayor accesibilidad no tiene su mejor ejemplo con las localidad de Vegas de Itata que en la realidad solo se comunica con la localidad de Perales y está unida al resto de la red por un solo camino.

El cálculo del Índice de Shimbel arrojó como resultado que las localidades de Coelemu (281), Quirihue (335) y Treguaco (326), todas ellas cabeceras comunales, son las más accesibles de toda la red. Continúa apareciendo eso si la distorsión de Vegas de Itata (325) como elemento de error en el cuadro general de aplicación del modelo. En cuanto a la accesibilidad de la red, el Índice de Dispersión (20.507) muestra que la red cuenta con una baja accesibilidad, lo que se corrobora con el Índice de Accesibilidad Media (354). Por último el Índice Omega confirma la información descrita, entregando como resultado que la localidad de Coelemu y la de Chancal representan los nodos con mayor y menor accesibilidad, respectivamente. 


\section{IV.4 Accesibilidad Absoluta y Relativa}
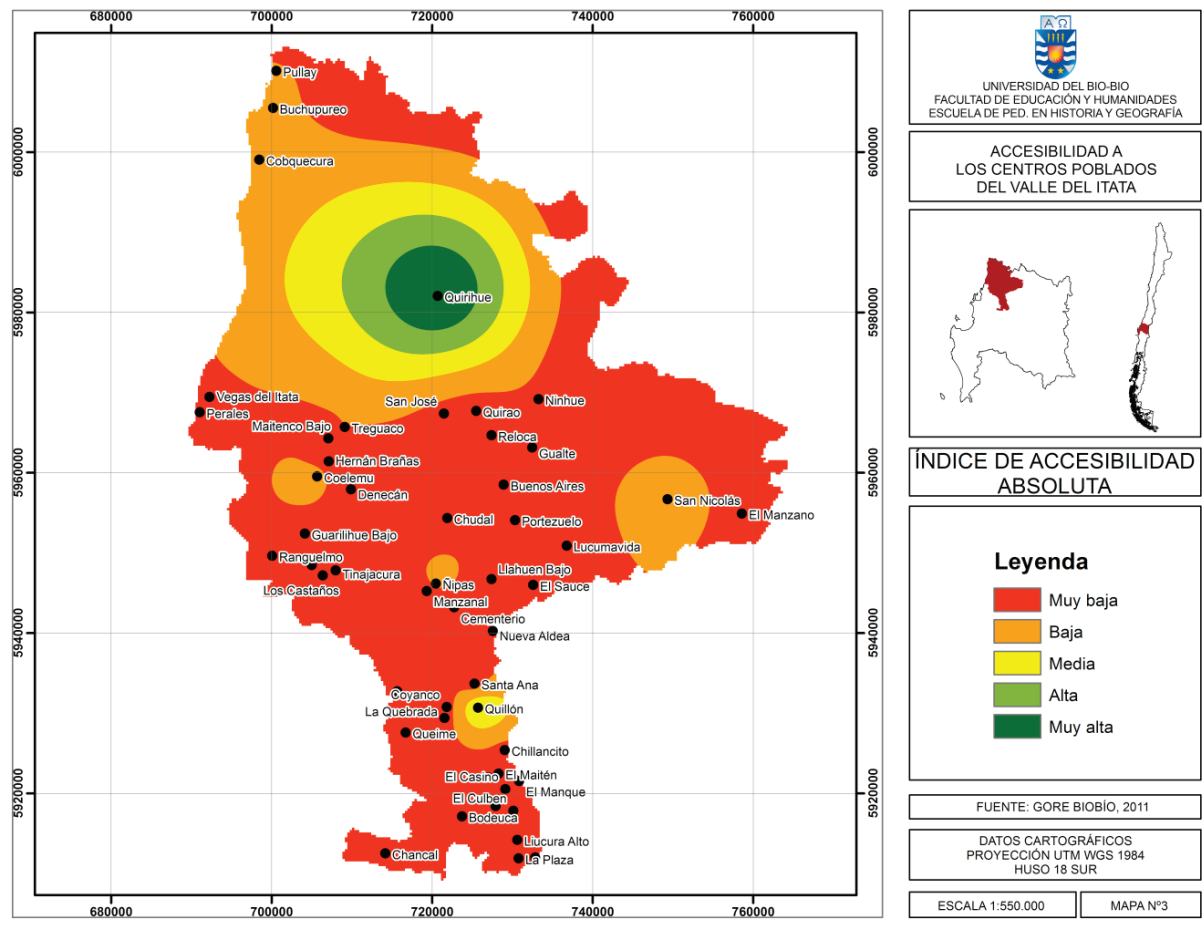

Con el cálculo de ambos indicadores se terminan confirmando los resultados presentados, sin embargo por las características de centralidad en el territorio es Quirihue y Quillón ambas cabeceras comunales, las localidades con mayores índices de accesibilidad; muy cerca de ellas se encuentra Coelemu desplazada a un tercer lugar. Las tres representan grados de importancia por estar estructuradas una en el centro del territorio (Quirihue), la otra por ser vaso comunicante con la costa (Coelemu) y la última por estar incorporada dentro de los circuitos de producción vitivinícola del país (Quillón).

En comparación, otras comunas del territorio con características similares tienen bajos índices de accesibilidad, tales como Cobquecura que siendo una zona turística y con arquitectura patrimonial se encuentra en el extremo norte, o la comuna de Portezuelo que estando relativamente central en el territorio y vinculada a la producción vitivinícola, se encuentra poco conectada por la presencia de cursos de agua y estructuras acolinadas. 

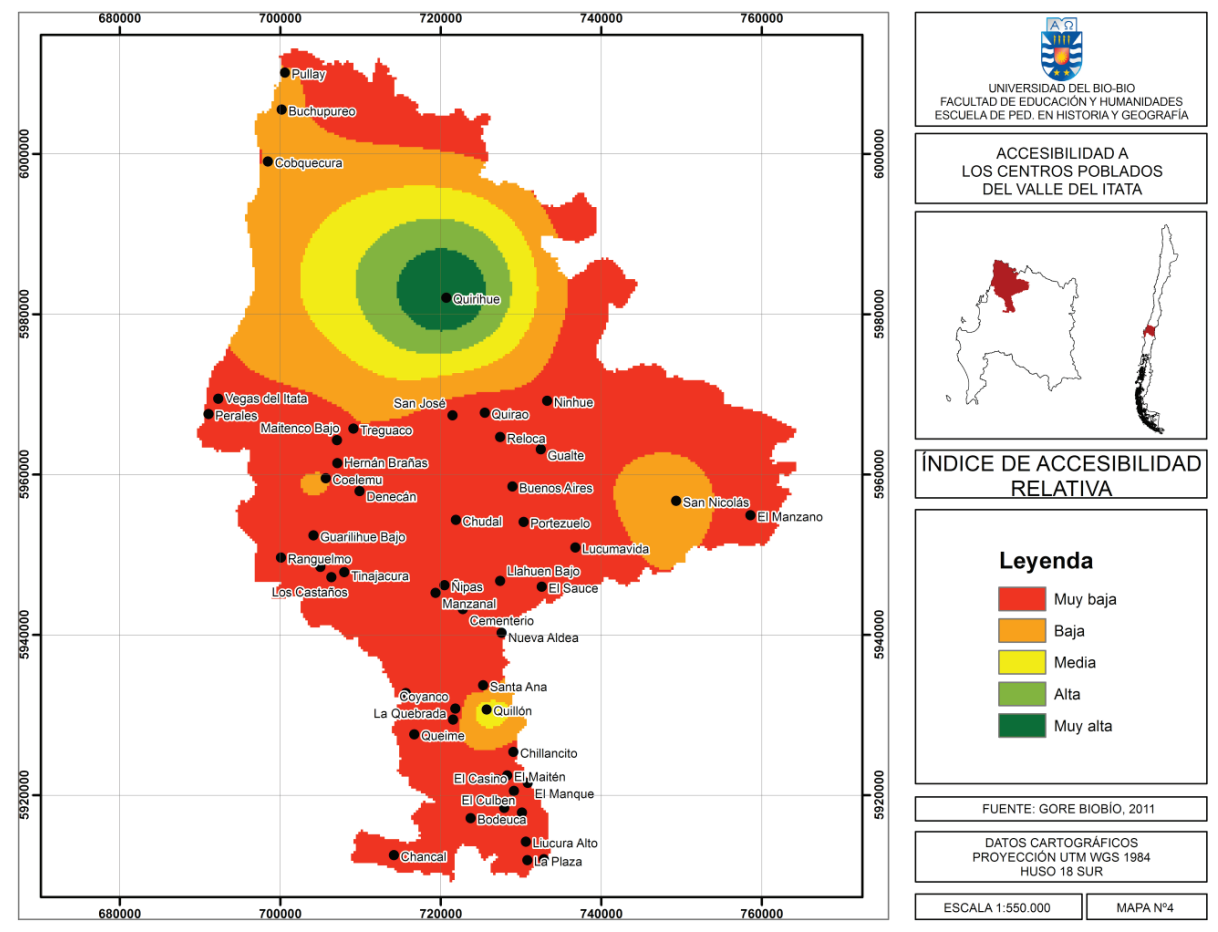

Asimismo se observa que la conectividad de los sectores costeros presentan restricciones entre los nodos pertenecientes a las comunas al norte del río Itata, con los nodos pertenecientes al sur del mismo, al no existir una estructura que mantenga la continuidad de las vías costeras, lo que implica que la comuna de Coelemu se transforme en un paso obligado que perdería relevancia al existir un puente sobre el rio Itata en la línea de costa, permitiendo disminución en los tiempos de desplazamiento y ahorro en recursos, consecuentemente estas zonas quedarían mucho más conectadas con la capital regional (Concepción) de lo que están ahora con Chillán.

\section{CONCLUSIONES}

El análisis de accesibilidad de los centros poblados del Valle del Itata no escapa a características comunes de accesibilidad que presentan a diferente escala, los países latinoamericanos. Una red de infraestructura vial frágil y desequilibrada, con zonas rurales intermedias conectadas por vías de tercer orden, 
de generación espontánea que confluyen en la red principal en algunos casos sin control ninguno. Se evidencia la necesidad de contar con nuevos criterios espacio-temporales de evaluación, tales como: el carácter económico, el estético, el turístico y otros, en algunos casos complementarios para evaluar la importancia de cada uno de los componentes con los que se busca explicar la accesibilidad.

En el actual caso la aplicación de indicadores de accesibilidad en un sistema de información geográfica permitió caracterizar el establecimiento de zonas con mayores y menores niveles de accesibilidad, que podrían corresponder a espacios de mayor desarrollo y a zonas desfavorecidas, al mismo tiempo permite la posibilidad de simular el territorio frente a modificaciones o propuestas, facilitando el análisis de incorporación de cambios en la dinámica espacial para el potenciamiento de áreas con menores índices de accesibilidad.

Esto porque la satisfacción de las necesidades suponen la interconexión de lugares geográficos mediante redes de transporte necesarios para los múltiples intercambios producidos entre los nodos, creando una red capaz de convertirse en uno de los principales factores explicativos de las diferencias de accesibilidad en el espacio. La distribución de los nodos ofrece una característica observable, que es la discontinuidad por la influencia de la geomorfología del territorio (Cordillera de la Costa).

La integración del territorio y sus centros poblados representa un amplio desafío por su envergadura, ya que la estructura vial aunque parece conectar las cabeceras comunales, en realidad demuestra que la concentración vial sirve como alimentador de cabeceras provinciales y regionales cuando la red se ve inserta en territorios más amplios, en desmedro del desarrollo vial del territorio, necesario para que se pueda cubrir y satisfacer las demanda de bienes y servicios, que se refleja en sectores alejados que presentan localidades con difíciles condiciones de vida, fuertes índices de pobreza y limitada productividad. Asimismo los resultados tienen correspondencia con la realidad ya que las localidades de Coelemu, Quirihue y Quillón, se han vuelto polos de atracción dentro de la red, agrupando servicios básicos de salud, trabajo, turismo y producción lo que fomenta el mantenimiento de las rutas viales. 


\section{BIBLIOGRAFÍA}

Alvarado, M. (2003). El vino en la historia de Chile y el mundo. (1 ${ }^{\text {a }}$ Edición) Santiago, Chile: Origo Ediciones.

Álvarez, C. (2001): Derecho del Vino. Denominaciones de Origen. ( $1^{\text {a }}$ Edición) Santiago, Chile: Editorial Jurídica de Chile.

Ávila, M. (2012): Políticas Públicas y Articulación del Territorio: Desarrollo de la Red Vial en la Región de Aysén. Memoria para optar al título Profesional de Geógrafo. Universidad de Chile, Facultad de Arquitectura y Urbanismo, Escuela de Geografía. Santiago - Chile. Recuperado en agosto de 2014 de http://www.tesis.uchile.cl/tesis/ uchile/2012/aq-avila_m/pdfAmont/aq-avila_m.pdf.

Bosque, J. (1997): Sistemas de Información Geográfica. (2 ${ }^{\mathrm{a}}$. ed.). Madrid. Ediciones Rialp, S.A.

Cardozo, O.; Gómez, E.; Parras, M. (2009): "Teoría de Grafos y Sistemas de Información Geográfica aplicados al Transporte Público de Pasajeros en Resistencia”. Revista Transporte y Territorio, No 1 Univ. de Bs. As, 89-111. Recuperado en agosto de 2014 de http://www.rtt.filo.uba.ar/RTT00105089.pdf.

Carrera, C.; Del Canto, C.; Gutiérrez, R; Méndez, R. y Pérez, M.C. (1988) Trabajos prácticos de geografía humana. Madrid. Editorial Síntesis.

Cepal (2012): Perfiles de infraestructura y Transporte en América Latina. Caso de Chile. Unidad de Servicios de Infraestructura de la División de Recursos Naturales e Infraestructura, de la Comisión Económica para América Latina y el caribe. Consultado en octubre de 2014 de http://www.cepal.org/perfil/noticias/noticias/7/29957/Caso_Chile.pdf.

Couyoumdian, J. (2006): Vinos en Chile desde la independencia hasta el fin de la belle époque (Historia, UC) Vol. 39 n.1 Santiago, Chile.

Deichman, U. (1997): Accessibility indicators in GIS. Department for Economic and Social Information and Policy Analysis. United Nations Statistics Division.

Del Pozo, J. (1999): Historia del vino chileno. Santiago, Chile: Editorial Universitaria.

Farrow, A. y Nelson, A. (2001): Modelación de la Accesibilidad en ArcView. Recuperado el 25 de marzo de 2008 de http:/www.ciat.cgiar.org/access/acceso/index.htm. Disponible en: http://ciat-library.ciat.cgiar.org/articulos_ciat/ciat_access_es.pdf

GARrido, J. (1995): "La organización espacial de la red de carretera en Aragón. Aplicación de la metodología de la teoría de grafos". Geographicalia, n 32, 83-102. Recuperado en agosto de 2014 de http:/www.unizar.es/geografia/geographicalia/garridografos.pdf.

Garrison, W. L. y Marble, D. F. (1964): "Factor-analytic study of the connectivity of a transportation network", Papers of the Regional Science Association, Volume 12, Issue 1, 231-238

Goodall, B. (1987): Dictionary of Human Geography. Puffin Paperback. Tx. USA.

Gross, P. (1998): “Ordenamiento territorial: el manejo de los espacios rurales" Eure, Diciembre Vol 24/ No 073, 116-118. Pontificia Universidad Católica. Santiago Chile. 
Recuperado el 25 de mayo de 2008 de http://www.scielo.cl/scielo.php?script=sci_artt ext\&pid=S0250-71611998007300006.

Gutiérrez, J.; MonZÓN, A. (1993): “La accesibilidad a los centros de actividad económica antes y después del Plan Director de Infraestructuras". Ciudad y territorio: Estudios territoriales, $\mathrm{N}^{\circ}$ 97, 385-395.

Hagget, P. y Chorley, P. (1969): Network Analysis in Geography. London: Arnold

Henríquez, F. M. (1990): "Climatología de la cuenca del río Itata. Santiago, Chile”, IGM, Revista. Geográfica de Chile Terra Australis, No 33, 7-27.

Henríquez, F. M. (1994): “Condiciones físicas y químicas de los suelos de Quirihue, Ninhue, Portezuelo, Ranquil, Chillán, Chile”, Tiempo y Espacio, año 4, n 4, 37-60.

Henríquez, F. M.; Olivares, P.; Ihl, M. (1997): "Posibilidades y Restricciones de la modernización agrícola y forestal del secano costero Provincia de Nuble Santiago, Chile" IGM, Revista Geográfica de Chile Terra Australis, No 42, 23-50.

Insaurralde, A.; Cardozo, O. (2010): Análisis de la Red Vial de la Provincia de Corrientes con Teoría de Grafos y Sistemas de Información Geográfica. Recuperado el 28 de abril de 2014 de http://www.rtt.filo.uba.ar/RTT00105089.pdf

LinNeKer, B. J. y SPEnce N., A. (1992): “An accessibility analysis of the impact of the M25 London Orbital Motorway on Britain”, Reg. Studies, 26, 31-47

LiRA, L. (2006): Ordenamiento del territorio. Segundo curso internacional sobre desarrollo local y competitividad territorial. ILPES-CEPAL, Naciones Unidas [en línea]

López, E., Gutiérrez, J. y Gómez, G. (2008): "Measuring regional cohesion effects of large-scale transport infrastructure investments: an accessibility approach". European Planning Studies, 16 (2), 277-301.

Martínez SÁnChez-Mateos, H.S. (2012): "La accesibilidad regional y el efecto territorial de las infraestructuras de transporte aplicación en Castilla-La Mancha". Boletín de la Asociación de Geógrafos Españoles, n ${ }^{\circ}$ 59, 79-103. Recuperado el 18 de julio de 2014 de http://dialnet.unirioja.es/servlet/articulo?codigo $=3938147$

Mop (2004): Diagnóstico y clasificación de los cursos y cuerpos de agua según objetivos de calidad cuenca del río Itata. Diciembre consultores de Ingeniería.

NASTAGI, N. (2003): “Accesibilidad viaria y desarrollo territorial en la España peninsular una propuesta de análisis". Geographicalia, 43, 81-96. Recuperado el 15 septiembre de 2014 de http://dialnet.unirioja.es/servlet/articulo?codigo $=650470$

Nogales, J.; Figueira, J.; Gutiérrez, J.; Pérez, P.; Cortés, T. (2002): “Determinación de la accesibilidad a los centros de actividad económica de Extremadura mediante técnicas SIG”. XIV Congreso Internacional de Ingeniería Gráfica, Santander (España). Recuperado el 10 de abril de 2008 de la base de datos INGEGRAF.

Pablo, F.; MuÑoz, C.; Myro, R. (2002): Un análisis del efecto de la inversión en infraestructuras sobre la accesibilidad del territorio a la red viaria de alta capacidad. Universidad de Alcalá, Dpto. de Estadística, Estructura Económica y O.E.I. Facultad de CC. Económicas y Empresariales, Alcalá de Henares Madrid

Potrykowsky, M. y TAylor, Z. (1984): Geografía del Transporte. Editorial Ariel, Barcelona.

Ramírez, M. (2003): “Cálculo de medidas de accesibilidad geográfica temporal y económica generadas mediante sistemas de información geográfica”. Primer Congreso 
de la ciencia cartográfica y VIII semana de cartografia, Buenos Aires (Argentina). Recuperado el 15 de abril de 2008, de http://www.elagrimensor.com.ar/elearning/lecturas/localiz.pdf

Rebolledo, J.; MuÑoz, J.; Henríquez, M. (1994): “Evaluación de la estabilidad morfogenética del secano costero de la provincia de Ñuble Chillán, Chile", Revista Tiempo y Espacio año $4 \mathrm{n}^{\circ} 4$; 61-77

RodríGUEZ, A. (2006). "VRP-XML: lenguaje de marcas extensible para los problemas de rutas de vehículos". X Congreso de Ingeniería de Organización, Valencia.

SALADO, M. (2004): "Localización de los equipamientos colectivos, accesibilidad y bienestar social”. En Sistemas de Información Geográfica y localización de instalaciones y equipamientos, Bosque, J. y Moreno, A. (1 $1^{\mathrm{a}}$. ed., 17-46). Madrid: Ra-Ma Editorial.

Seguí, J. y Martínez, M. (2003): "Pluralidad de métodos y renovación conceptual en la geografía de los transportes del siglo XXI". Scripta Nova, vol. VII, 139. Recuperado el 27 de abril de 2008, de http://www.ub.es/geocrit/sn/sn-139.htm \}

Seguí Pons, J.M.; Petrus Bey, J.M. (1991): Geografía de redes y sistemas de transportes, Madrid, Síntesis.

TINKLER, K. (1977): An introduction to graph theoretical methods in geography. Geo Abstracts Ltd. 